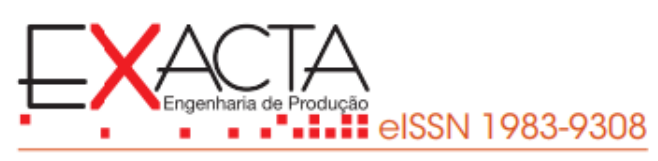

\title{
INTERNET DAS COISAS EM SISTEMAS LOGÍSTICOS: REVISÃO DA LITERATURA RECENTE E PERSPECTIVAS DE PESQUISA
}

\section{INTERNET OF THINGS IN LOGISTICS SYSTEMS: RECENT LITERATURE REVIEW AND RESEARCH PERSPECTIVES}

Versão do autor aceita publicada online: 19 fev. 2020

Publicado online: 12 maio 2021

Como citar esse artigo - American Psychological Association (APA):

Agostino, Í. R. S., Ristow, C., \& Rodriguez, C. M. T. (2021, abr./jun.). Internet das coisas em sistemas logísticos: revisão da literatura recente e perspectivas de pesquisa. Exacta, 19(2), 251-275. https://doi.org/10.5585/exactaep.2021.15999.

Submeta seu artigo para este periódico $\beta$

Dados Crossmark 


\title{
INTERNET DAS COISAS EM SISTEMAS LOGÍSTICOS: REVISÃO DA LITERATURA RECENTE E PERSPECTIVAS DE PESQUISA
}

\author{
INTERNET OF THINGS IN LOGISTICS SYSTEMS: RECENT LITERATURE REVIEW AND \\ RESEARCH PERSPECTIVES
}

\section{Dícaro Romolo Sousa Agostino ${ }^{1}$ \\ iD Charles Ristow ${ }^{2}$ \\ iD Carlos Manuel Taboada Rodriguez ${ }^{3}$}

\author{
${ }^{1}$ Mestre \\ Universidade Federal de Santa Catarina - UFSC \\ icaroagostino@gmail.com \\ 2 Mestre \\ Universidade Federal de Santa Catarina - UFSC \\ charles.ristow@gmail.com \\ ${ }^{3}$ Doutor \\ Universidade Federal de Santa Catarina - UFSC \\ carlos.taboada@ufsc.br
}

Recebido em: 07 nov. 2019

Aprovado em: 19 fev. 2020
Resumo: Este artigo tem como objetivo apresentar perspectivas para aplicação de tecnologias loT em sistemas logísticos cobrindo aspectos teóricos e práticos da área de pesquisa, além de fornecer um portfólio bibliográfico atualizado de estudos que relacionam a temática. Foi realizada uma Revisão Sistemática de Literatura objetivando identificar as principais características da área de investigação e de agrupar os estudos teóricos e as perspectivas práticas analisadas. Como resultados, a análise bibliométrica evidenciou o crescimento da área de pesquisa e das revistas científicas mais importantes que publicam conteúdo relacionado a plataformas baseadas em loT em contextos logísticos. Na análise de conteúdo, as perspectivas são agrupadas em: (i) proposições e requisitos conceituais, (ii) novos métodos e modelos de apoio à tomada de decisão, (iii) desenvolvimento de abordagens de base tecnológica e (iv) estudos empíricos. Como conclusão, é apresentado a descrição de direções para perspectivas futuras, tanto do ponto de vista científico quanto do ponto de vista prático.

Palavras-chave: Sistemas logísticos. Indústria 4.0. Internet das Coisas (IoT). Revisão sistemática de literatura.

Abstract: This paper aims to present perspectives for the application of loT technologies in logistics systems covering theoretical and practical aspects of the research area, in addition to providing an updated bibliographic portfolio of studies that relate to the theme. A Systematic Literature Review was carried out in order to identify the main characteristics of the research area and to group the theoretical studies and the practical perspectives analyzed. As results, the bibliometric analysis evidenced the growth of the research area and the most important scientific journals that publish content related to loT-based platforms in logistics contexts. In content analysis, the perspectives are grouped into: (i) conceptual propositions and requirements, (ii) new methods and models to support decision making, (iii) development of technology-based approaches and (iv) empirical studies. In conclusion, is presented the description of directions for future perspectives, both from the scientific point of view and from the practical point of view.

Keywords: Logistics systems. Industry 4.0. Internet of Things (IoT). Systematic literature review. 


\section{Introdução}

Sistemas logísticos envolvem a gestão de fluxos de bens, serviços, finanças e informações, com foco no desempenho da cadeia de suprimentos, comumente apresentando características complexas e dinâmicas (Haq \& Kannan, 2006; Lima-Junior \& Carpinetti, 2019). Nesse contexto, as cadeias de suprimentos são definidas como um sistema que interliga diversos processos, desde a movimentação de matérias-primas e insumos, fabricação, até o usuário final (Haq \& Kannan, 2006).

Por isso, muitas organizações direcionam esforços para responder de forma mais eficaz às exigências logísticas (Lee, Jung, Eum, Park, \& Nam, 2006; Silva, Ferreira, Silva, Magalhães, \& Neto, 2017). Atividades como a controle de operações em tempo real, monitoramento de nível de serviço e sensoriamento remoto são essenciais para o aumento da produtividade e da qualidade em diversas funções organizacionais, influenciando os processos de tomada de decisões, a gestão e avaliação do desempenho empresarial (Haq \& Kannan, 2006).

Avanços tecnológicos como Internet of Things (IOT), big data e inteligência artificial aumentaram a disponibilidade e o volume de dados em sistemas de produção (Tao, Qi, Liu, \& Kusiak, 2018) reduzindo o custo de coleta e armazenamento de grandes conjuntos de informações (Peres \& Fogliatto, 2018), tecnologias loT proveem tais capacidades estendendo a interconexão entre diversos dispositivos ligados a processos industriais e logísticos, permitindo a coleta de dados de forma intermitente, orientando a tomada de decisão síncrona ao considerar diversos aspectos do estado atual de sistemas produtivos (Qu, et al., 2016; Tao, Qi, Liu, \& Kusiak, 2018).

O aumento do uso de sensores em máquinas e equipamentos, frequentemente referido como “Indústria 4.0" (Lee, Bagheri, \& Kao, 2015; Wang, Törngren, \& Onori, 2015; Monostori, et al., 2016) tem viabilizado o desenvolvimento de abordagens baseadas em dados em tempo real, suportada pelo crescente poder computacional, com foco em controle adaptativo de processos (Frazzon, Kück, \& Freitag, 2018).

O termo "Internet das Coisas", do inglês Internet of Things (IoT), pode ser definido como uma infraestrutura capaz de realizar interconexão entre sistemas físicos e virtuais, por meio de tecnologias de informação e comunicação de diversos dispositivos ligados a internet (Ray, 2016; Mahdavinejad, et al., 2018). Thoben, Wiesner e Wuest (2017) argumentam que um dos principais aspectos da quarta revolução industrial está ligada a incorporação de tecnologias loT em sistemas de produção, permitindo integração horizontal e vertical dos diversos processos industriais, em que dados são coletados e armazenados ao longo da produção, assim como da cadeias de suprimentos, viabilizando a construção de uma plataforma para tomada de decisão orientada a reações rápidas e execuções de mudanças no ambiente industrial (Tao, Qi, Liu, \& Kusiak, 2018; Frazzon, Kück, \& Freitag, 2018). 
Tais abordagens baseadas em informações em tempo real podem prover plataformas de alta qualidade para tomadores de decisões (Heger, Grundstein, \& Freitag, 2017), existindo uma necessidade crescente de desenvolver estudos de viabilidade para a implementação de plataformas loT em sistemas de produção e logística (Leusin, Frazzon, Maldonado, Kück, \& Freitag, 2018; Qu, et al., 2016). Essas plataformas aparecem como infraestrutura necessária para a tomada de decisão integrada e sincronizada em sistemas distribuídos e automatizados (Avventuroso, Silvestri, \& Frazzon, 2018).

Alguns estudos de revisão anteriores abordam loT no contexto de sistemas logísticos, Aryal, Liao, Nattuthurai e Li (2018) revisaram a literatura sobre aplicações de tecnologias loT, big data e análise de dados em cadeias de suprimento, verificando o crescente interesse na temática e os potenciais de aplicações com foco em integração de cadeias de suprimento e análise de dados em tempo real. Em outro estudo correlato Qu et al. (2016) investigaram a aplicabilidade de loT em sistemas de produção e logística com foco na sincronização de informações em tempo real, permitindo maior flexibilidade e controle dinâmico de sistemas integrados, os autores desenvolveram e aplicaram uma abordagem loT em um caso industrial real de larga escala, encontrando resultados positivos no aumento da produtividade do sistema.

Como contribuição original a literatura, este artigo apresenta o estado da atual das aplicações de tecnologias loT em sistemas logísticos. De forma objetiva este artigo apresenta as seguintes contribuições: (i) Apresenta um portfólio de artigos atualizados e com um recorte temático mais especifico, não considerando tecnologias complementares, com foco em loT e suas aplicações em sistemas logísticos; (ii) Caracteriza o campo de pesquisa identificando principais veículos de comunicação, evolução temporal, principais autores e outros aspectos do estado atual da área; (iii) identifica lacunas e oportunidades de pesquisa a serem exploradas, fornecendo para pesquisadores da área um panorama geral das pesquisas e do estado da arte.

Diante do exposto, esse trabalho objetiva conhecer a literatura recente sobre loT no contexto logístico, para tanto foi conduzida uma Revisão Sistemática da Literatura (RSL), dessa forma as seguintes perguntas de pesquisa nortearam o desenvolvimento desta pesquisa: (i) qual a evolução temporal, principais países e periódicos que vinculam pesquisas envolvendo loT em sistemas logísticos? (ii) qual os temas importantes para pesquisas envolvendo loT em logística? (iii) quais as perspectivas de pesquisa e qual o atual estado de desenvolvimento da área?

Além dessa introdução, o artigo está estruturado da seguinte forma: na seção 2 é apresentada a abordagem metodológica adotada, assim como os critérios de seleção e inclusão dos estudos no processo de revisão; na seção 3 são apresentados os resultados na forma de uma análise bibliométrica e de conteúdo dos estudos analisados; por fim, na seção 5 apresentam-se as conclusões e as oportunidades de pesquisa futuras. 


\section{Abordagem metodológica}

Com o objetivo de conhecer, analisar e avaliar a literatura atual sobre loT em Sistemas Logísticos foi realizada uma revisão sistemática da literatura (RSL) sobre a temática, guiada pelas questões definidas na seção 1. O processo de busca considerou as bases Web of Science e Scopus, sendo reconhecidas como os maiores repositórios de documentos científicos (Guerrero-Bote \& Moya-Anegón, 2012). Para tanto, foi construída uma estratégia de busca a partir da definição de termos centrais da área. A Tabela 1 apresenta as versões finais das strings de busca utilizadas em cada base, assim como a quantidade de resultados em termos do número de publicações. Foram consideradas nas bases apenas publicações de periódicos e em inglês.

Tabela 1 - Strings de busca

Database

Web of Science

Scopus

$$
\begin{aligned}
& \text { TITLE-ABS-KE } \\
& \text { ("Logistic*")) }
\end{aligned}
$$

Fonte: Elaborado pelo autor String de busca

\section{Resultados}

563

A busca foi realizada no mês de abril de 2019, foram encontrados 563 artigos, sendo 224 da base Web of Science e 339 da Scopus. Os termos utilizados para o primeiro constructo foram "Internet of Things" e "IoT" e para o segundo "logistic*" sendo a busca aplicada nos títulos, resumos e palavras chaves dos artigos. O protocolo de pesquisa foi então construído conforme o modelo de processo apresentado na Figura 1, o principal propósito desse estudo foi selecionar apenas artigos que claramente tratavam da aplicação de tecnologia loT em contextos Logísticos. 
Figura 1 - Processo de busca e seleção dos artigos

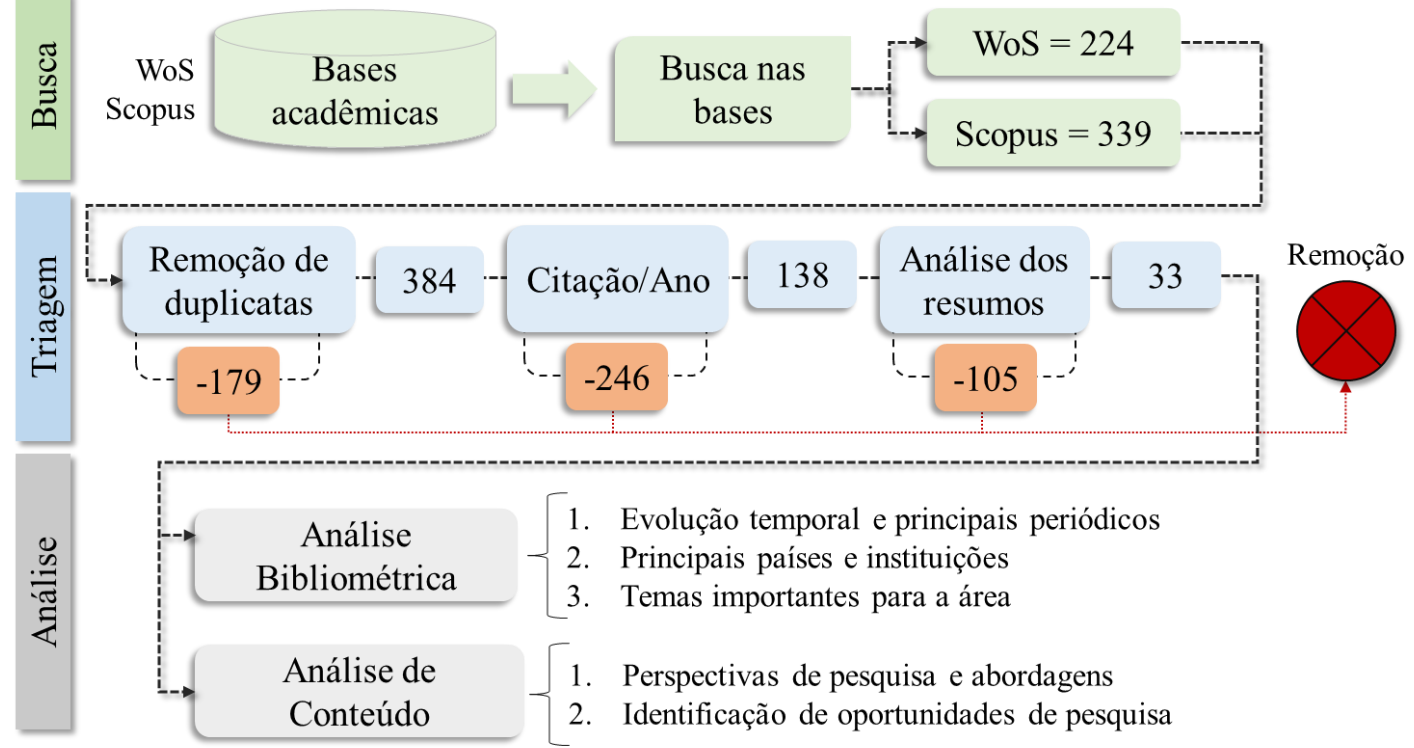

Fonte: Elaborado pelo autor.

A taxonomia de classificação composta de cinco macroetapas: (i) identificação do problema de pesquisa; (ii) a busca nas bases de periódicos; (iii) remoção de artigos duplicados; (iv) remoção das publicações fora do intervalo temporal compreendido entre 2014 e 2019 e remoção de artigos que não obtiveram no mínimo 3 citações; (v) leitura dos títulos e resumos para identificar o alinhamento com as questões de pesquisa. A seleção final totalizou 33 artigos publicados em periódicos com alinhamento a temática e a análise bibliométrica e de conteúdo foi realizada considerando esse grupo.

Como recurso computacional para manipulação e análise dos dados foi utilizado o software R 3.5.2 (R Core Team, 2018), em conjunto com a IDE RStudio 1.2.1335. Todas as análises foram suportadas pelo pacote "Bibliometrix" 2.1.2 (Aria \& Cuccurullo, 2017).

\section{Resultados}

Os resultados serão apresentados em duas seções: (i) análise bibliométrica, em que são investigadas as características do portfólio bibliográfico analisado; (ii) análise de conteúdo, com o objetivo de classificar perspectivas de pesquisa e identificar oportunidades futuras de pesquisa.

\subsection{Análise bibliométrica}

Na Figura 2 é ilustrada a evolução temporal das publicações no portfólio selecionado. Considerando o recorte temporal dos últimos cinco anos, é possível evidenciar o crescimento da área com tendência de positiva. Essa análise permite destacar o crescente interesse em aplicações baseadas em loT em sistemas logísticos considerando os critérios de seleção adotados. 
Figura 2 - Evolução temporal das publicações

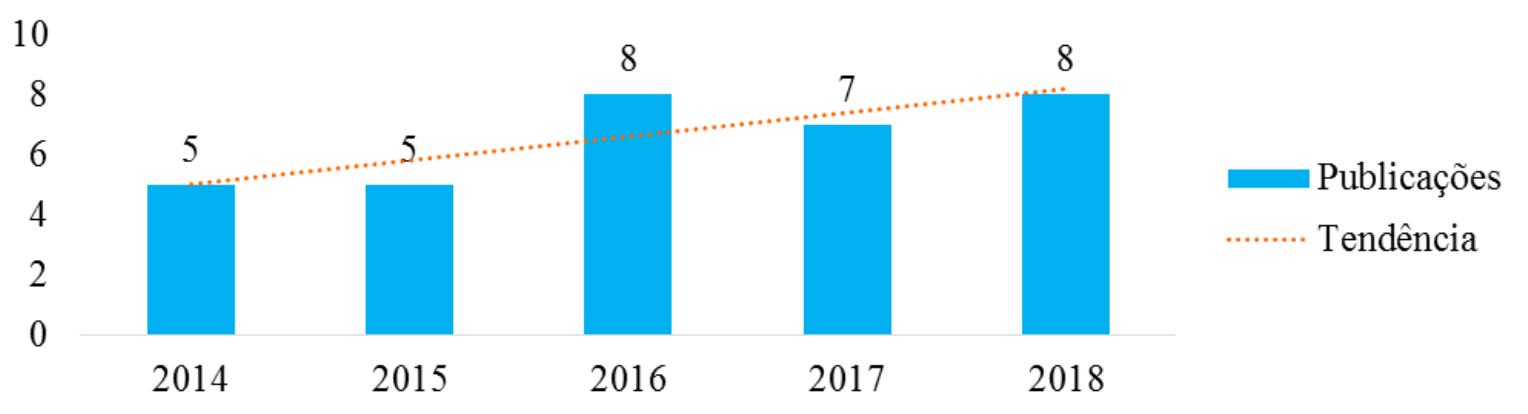

Fonte: Elaborado pelo autor

Na Figura 3(a) apresentam-se os dez periódicos que tiveram maior concentração de publicações no grupo analisado. O periódico "Industrial Manufacturing \& Dara Systems" foi o que apresentou maior número de publicações. Na Figura 3(b) são apresentados os dez periódicos que mais vincularam pesquisa que foram citados no grupo de estudos analisado, nesse caso observa-se grande destaque ao "International Journal of Production Economics" entre outros periódicos que vinculam estudos de gestão de operações, logística e tecnologia.

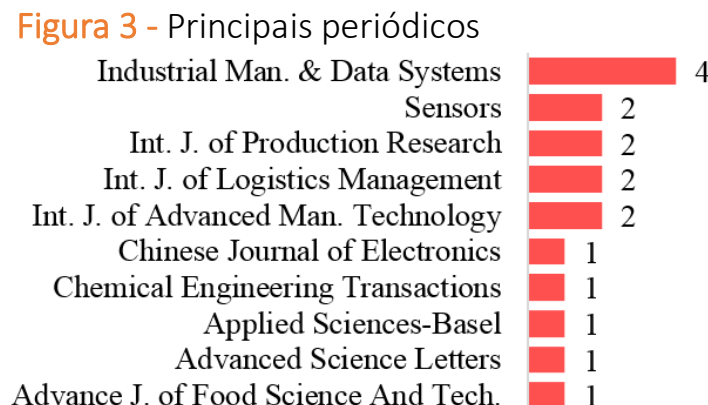

(a) Periódicos do grupo analisado Fonte: Elaborado pelo autor.
$4 \quad$ Int. J. of Production Economics IEEE Trans. on Industrial Informatics Int. J. of Production Research Int. J. of Advanced Man. Technology European J. of Operational Research Robotics and Comp. Integrated Man. Int. J. of Computer Integrated Man. Computers in Industry Computers \& Industrial Eng. Expert Systems with Applications

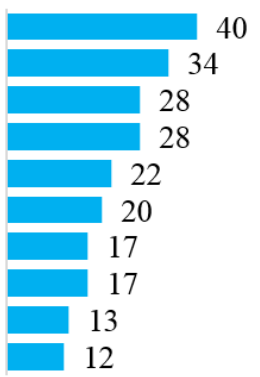

(b) Periódicos mais citados

A produção dos autores que contribuíram para a área é apresentada na Figura 4, listando os dez autores mais produtivos do portfólio bibliográfico analisado, relacionando o total de publicações com a quantidade de citações recebidas por cada autor. Entre os autores, George Q. Huang apresenta seis publicações, sendo duas em 2015, três em 2016 e uma em 2017, sendo o autor mais produtivo dentro do portfólio bibliográfico considerado. 
Figura 4 - Produção dos autores ao longo do tempo

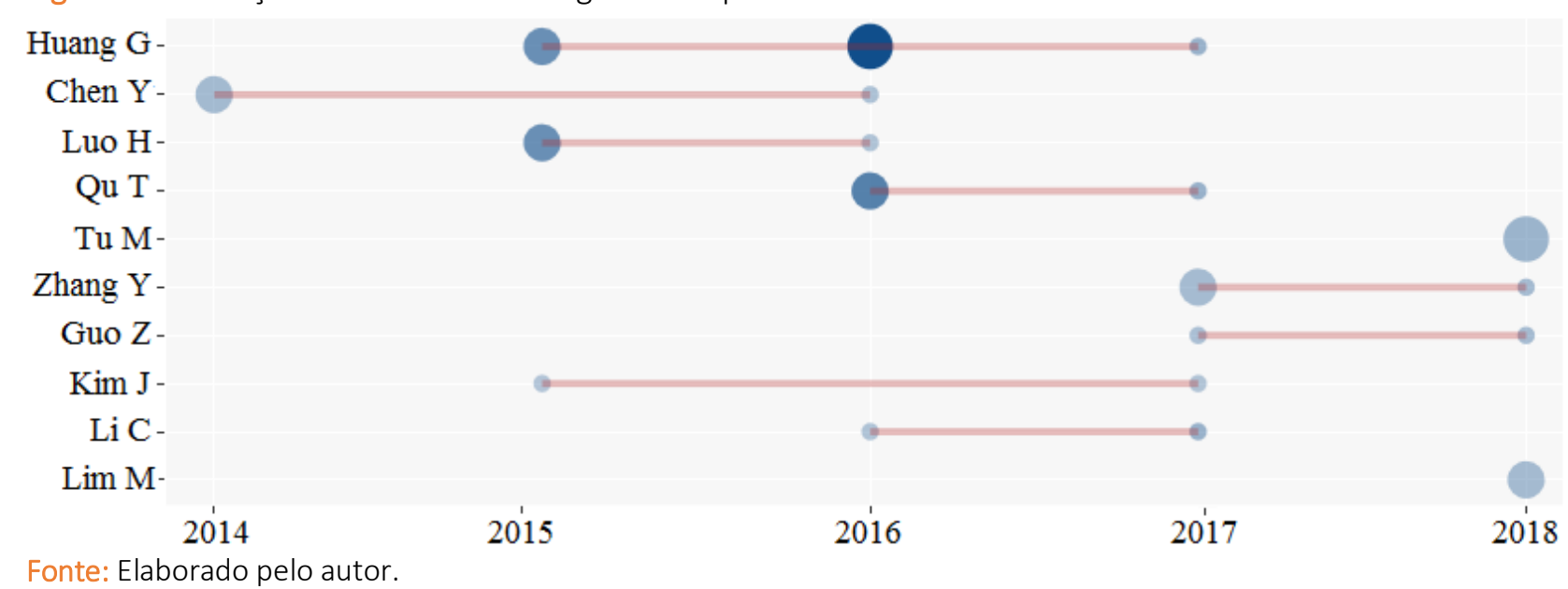

A distribuição geográfica das pesquisas é apresentada na Figura 5, sendo em azul os estudos vinculados a um único país, e em vermelho estudos em colaboração internacional. O país que mais vincula pesquisa na temática é China com 13 publicações, seguido de Taiwan com seis e Coreia do Sul com quatro, os demais apresentaram apenas um estudo cada no portfólio bibliográfico considerado.

Figura 5 - Quantidade de publicação por país

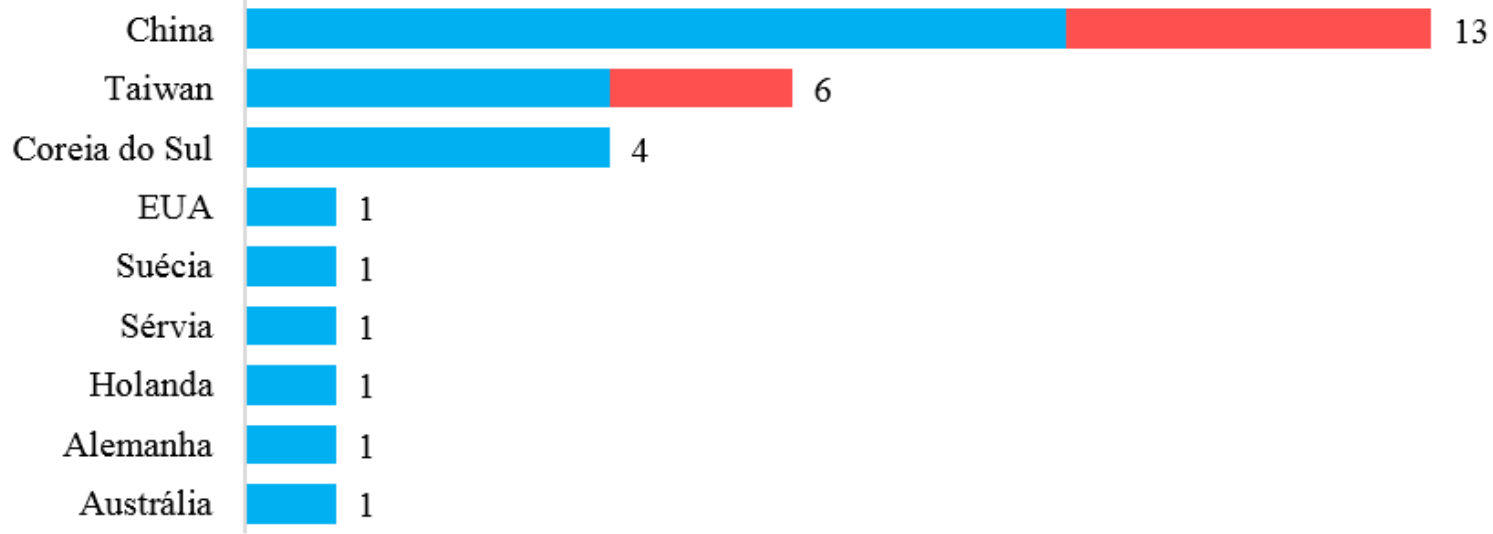

— Publicações de um único país = Publicações de vários países

Fonte: Elaborado pelo autor.

Na Figura 6 é apresentada a rede de cocitação a partir do algoritmo de Fruchterman e Reingold (1991). Dessa forma, percebe-se a formação de um grupo principal (em vermelho) formado pelos autores com maior ocorrência em termos de cocitação. Nesse grupo é possível identificar autores com importantes contribuições, com George Q. Huang já destacado anteriormente na análise da produção dos autores ao longo do tempo. O autor com maior centralidade foi Ting $Q u$, indicando forte influência na área de pesquisa para o período considerado, sendo que os trabalhos mais citados do autor grupo 
analisado voltados para integração da produção e logística por meio de tecnologias loT (Qu, et al., 2016; Thürer, et al., 2016; Qu, et al., 2017).

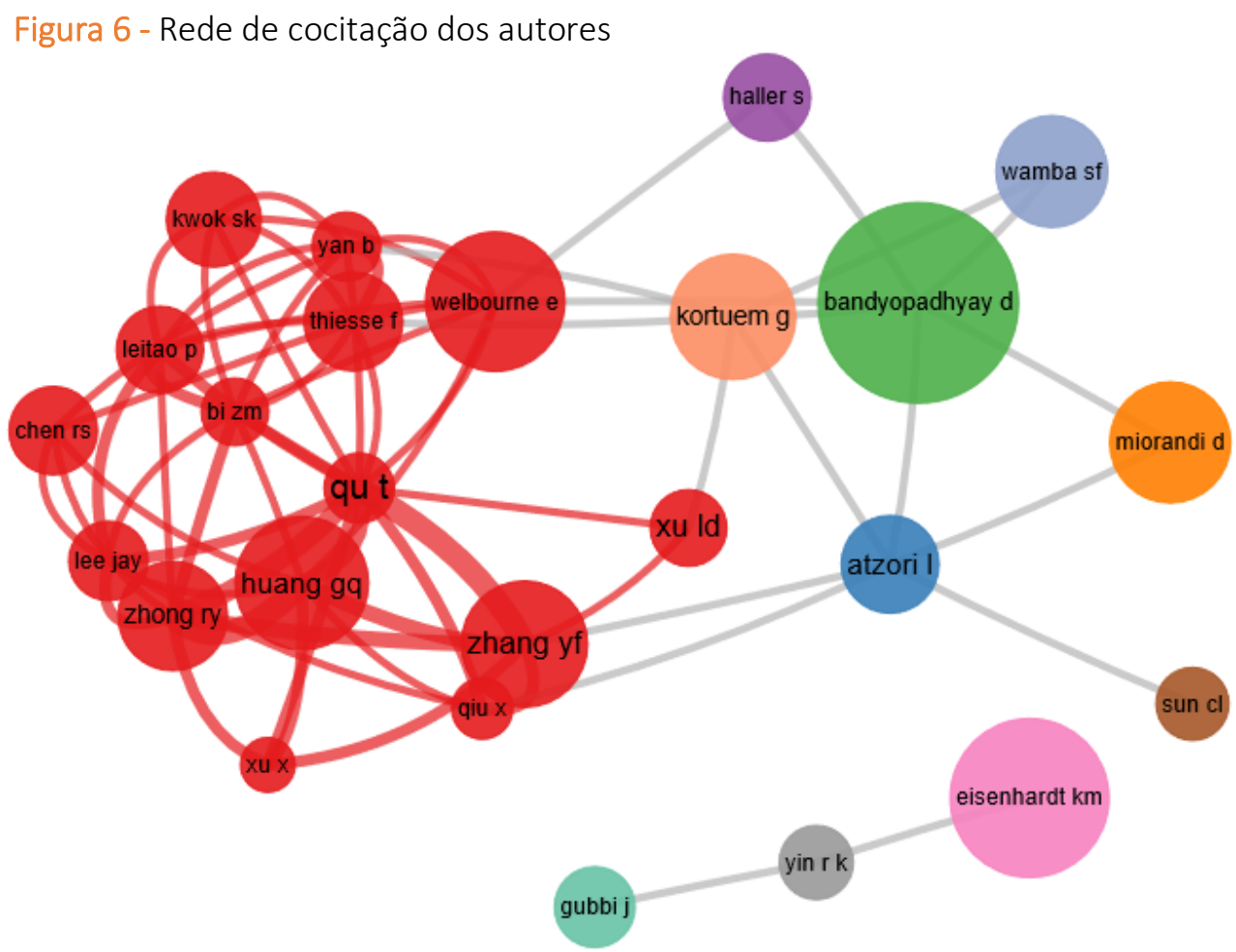

Fonte: Elaborado pelo autor.

Para identificação dos termos mais recorrentes foi utilizado uma de rede de coocorrência das palavras chaves dos artigos, utilizando o algoritmo de Fruchterman e Reingold (1991), conforme a Figura 7. Percebe-se que o grupo de publicações selecionado está alinhado ao tema a ser investigado, com o termo "internet of things" aparecendo como central. O termo "radio frequency identification" (RFID) indica o direcionamento das pesquisas para utilização dessa tecnologia em conjunto com sistemas logísticos. Também é possível destacar os termos "logistics" e "simulation", indicando a área de aplicação e a abordagem quantitativa mais recorrente, respectivamente. 
Figura 7 - Rede de palavras chaves dos artigos

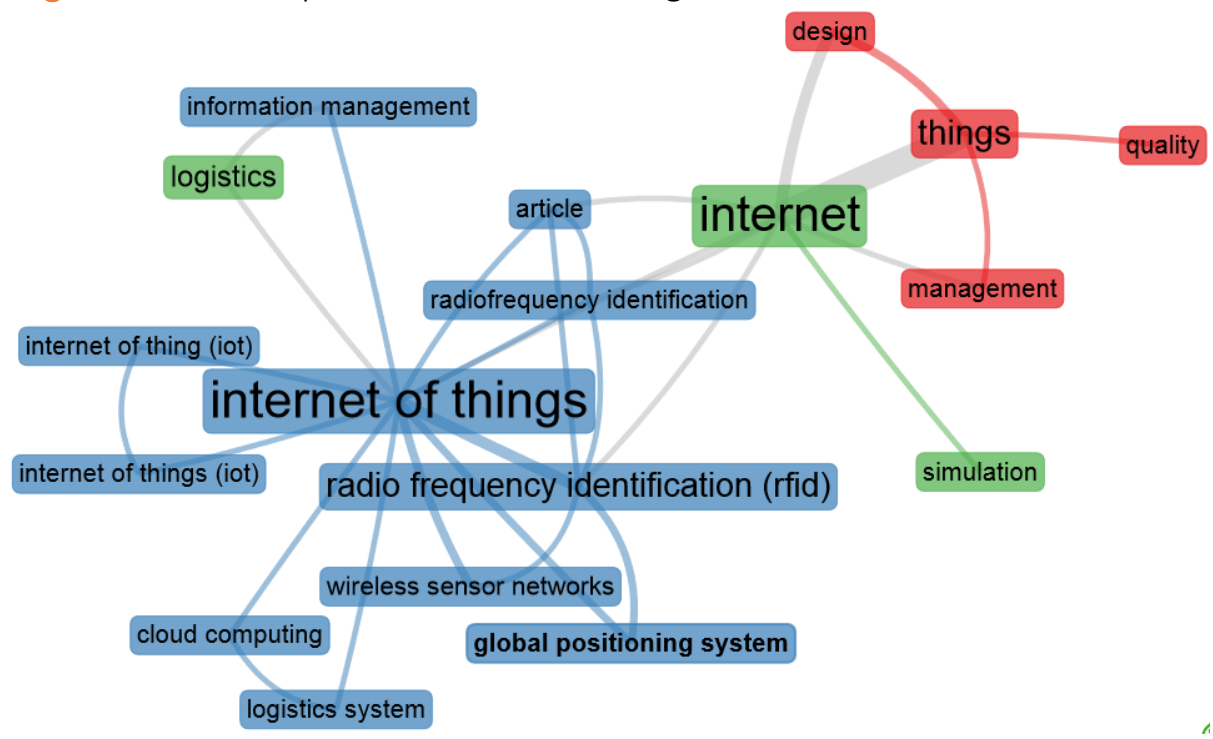

Fonte: Elaborado pelo autor.

Com o objetivo de realizar um mapeamento temático da área de estudo analisada, Cobo et al. (2011) sugere a construção de um mapa temático considerando os termos mais evidenciados nos títulos das publicações analisadas, relacionando a densidade e centralidade dos termos. Na Figura 8 ilustra-se a construção do mapa temático do grupo de estudos selecionados. Os temas localizados no quadrante superior direito representam temas motores, sendo áreas bem desenvolvidas e importantes para a estruturação de um campo de pesquisa, no caso estudado os termos internet e radio frequency identification aparecem nessa classificação. Os temas no quadrante superior esquerdo representam temas especializados, aparecendo o termo wireless sensor networks.

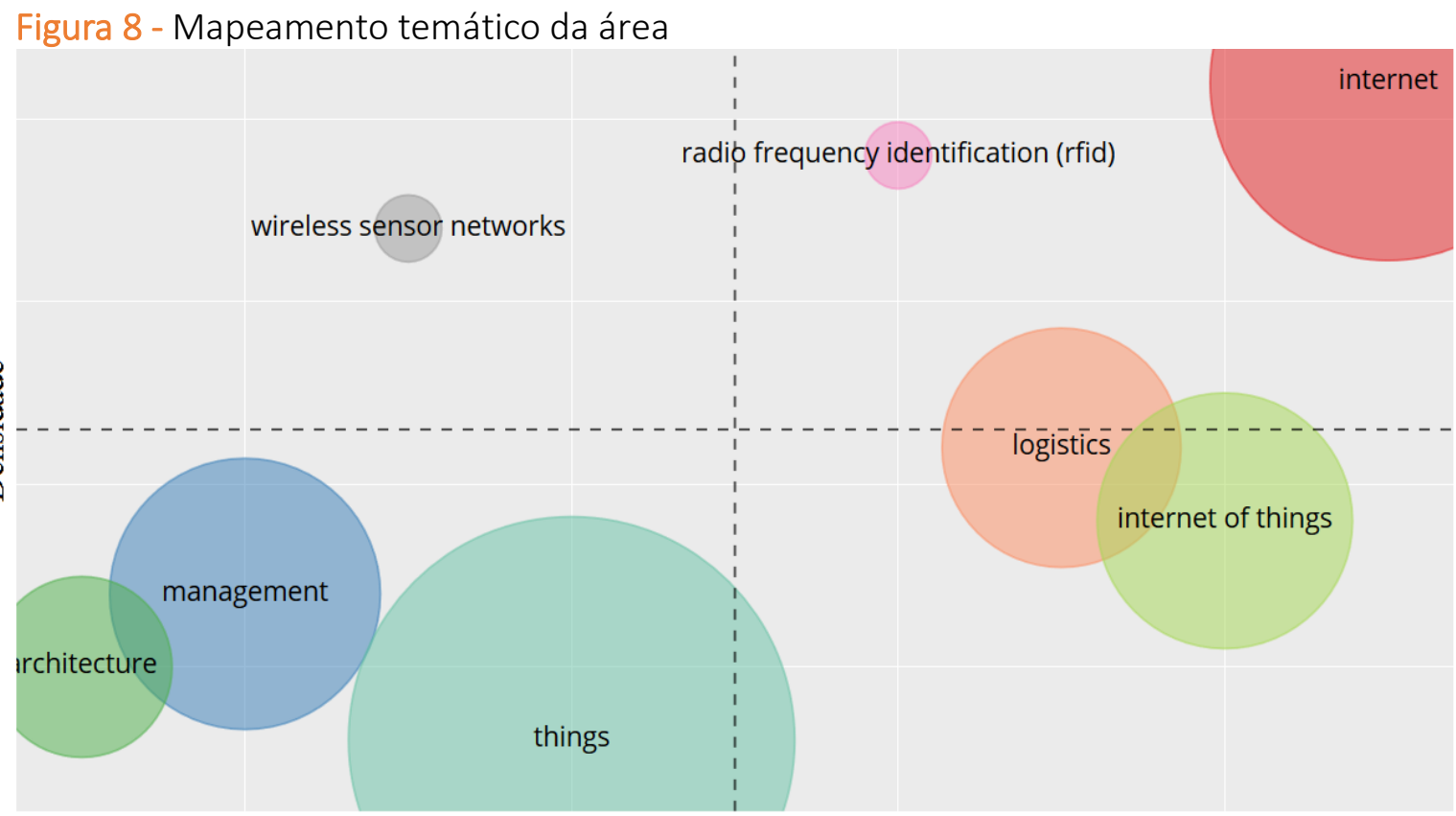

Fonte: Elaborado pelo autor

Centralidade 
No quadrante inferior esquerdo estão os temas emergentes, aparecendo architeture, management e thing. Por fim, no quadrante inferior direito estão os temas base, que são considerados importantes para o desenvolvimento do campo, sendo mais genéricos e transversais, os temas logistics e internet of things estão nessa categoria.

Na seção seguinte será analisado o conteúdo do portfólio bibliográfico considerado, abordando as características dos estudos com o objetivo de identificar perspectivas futuras de pesquisa.

\subsection{Analise de conteúdo}

Essa seção apresenta como resultados: um portfólio de estudos classificados por perspectivas, a análise da concentração de ocorrências em cada classe, a evolução das perspectivas ao longo do tempo, e por fim uma discussão de cada perspectiva a partir de evidências e tendências observadas nos estudos. A análise de conteúdo realizada (em apêndice) permitiu a classificação do portfólio bibliográfico em quatro perspectivas: (i) estudos teóricos e/ou conceituais, (ii) proposição de novos métodos e modelos para tomada de decisão, (iii) desenvolvimento de abordagens tecnológicas aplicadas e (iv) estudos empíricos. A Tabela 2 apresenta o portfólio de estudos classificados por perspectiva.

\section{Tabela 2 - Classificação dos estudos por perspectiva Classificação} Autor (ano)

(i) Estudos teóricos e/ou Tu, Lim e Yang (2018a), Qu et al. (2017), Maslarić, Nikoličić e Mirčetić (2016), Guo e Qu conceituais (2015), Zou, Chen, Uysal e Zheng (2014) Zhang, Guo, Lve Liu (2018), Lee, Lv, Ng, Ho e Choy (2018), Guo, Zhang, Zhao e Song

(ii) Proposição de novos (2017), Yan (2017), Trappey et al. (2017), Zhang, Zhao e Qian (2017), Cho e Kim (2017), métodos e modelos para Zhong, Lan, Xu, Dai e Huang (2016), Thürer et al. (2016), Yang, Li e Hu (2016), Qiu, Luo, tomada de decisão Xu, Zhong e Huang (2015), Kong, Fang, Luo e Huang (2015), Chen, Chen e Hsu (2014), He e Chu (2014)

(iii) Desenvolvimento de Verdouw, Robbemond, Verwaart, Wolfert e Beulens (2018), Accorsi, Cholette, Manzini e abordagens tecnológicas Tufano (2018), Chuang, et al. (2017), Kang, Park e Youm (2016), Li, Peng, Zhang, Wei e Li aplicadas (2015), Kim, Lee e Oh (2015), Chandra \& Lee (2014), Chen, Zhao e Zhai (2014), Tu (2018)

(iv) Estudos empíricos Tu (2018), Hopkins \& Hawking (2018), Tu, Lim e Yang (2018b), Qu et al. (2016), Papert, Rimpler e Pflaum (2016), Hu, Huang, Hou, Chen e Bulysheva (2016)

Fonte: Elaborado pelo autor.

Estudos teóricos e/ou conceituais apresentaram cerca de 15\% do total de publicações do portfólio, os estudos nessa classificação em sua maioria tratam de revisões de literatura e proposições 
de conceitos que relacionam a área de tecnologias loT com sistemas logísticos, apoiados na literatura recente da indústria 4.0.

A maior concentração de estudos ocorreu na perspectiva (ii), cerca de $42 \%$, se tratando de proposição de métodos, modelos e abordagens para gerenciar e/ou otimizar sistemas logísticas utilizando como base tecnologias loT. Esse grupo de estudos se caracteriza predominantemente pela proposição conceitual, em que parte das publicações apresentam experimentos numéricos simulados ou utilizam dados reais para testar abordagens, mas não apresentam aplicações diretas com evidências empíricas como resultados.

A perspectiva de desenvolvimento de abordagens tecnológicas considerou estudos que tratavam da análise e desenvolvimento de tecnologias envolvendo software e hardware para viabilização do sensoriamento e capacidades responsivas de dispositivos e sistemas baseados em loT. Essa classificação concentrou cerca de $24 \%$ das publicações, grande parte dos estudos nessa temática relacionavam tecnologias de Identificação por Radiofrequência (RFID) como forma de interconexão entre dispositivos do sistema.

Estudos que tratavam de aplicações com evidencias empíricas representaram cerca de $18 \%$ do portfólio analisado. Predominantemente foi observado estudos que relacionam características da indústria 4.0 com sistemas logísticos, explorando conceitos de sistemas ciber-físicos com interconexão ao longo de cadeias de suprimentos, com foco em maior controle e eficiência, assim como aproximação e integração da produção e logística. Todos os estudos nessa classificação relatam ganhos ao adotar tecnologias loT na logística.

A evolução das publicações por perspectivas propostas é apresentada na Figura 9, percebe-se um direcionamento recente para proposição de novos métodos e modelos em conjunto com estudos aplicados, indicando um amadurecimento da área com foco em avaliar a teoria desenvolvida em aplicações reais. As perspectivas (i) Estudos teóricos e/ou conceituais e (ii) Proposição de novos métodos e modelos para tomada de decisão não apresentou crescimento no período analisado, corroborando o amadurecimento da área para a direção da evidenciação dos resultados a partir da aplicação de tecnologias loT em contextos logísticos. 
Figura 9 - Perspectivas de aplicação de tecnologias loT em logística ao longo do tempo

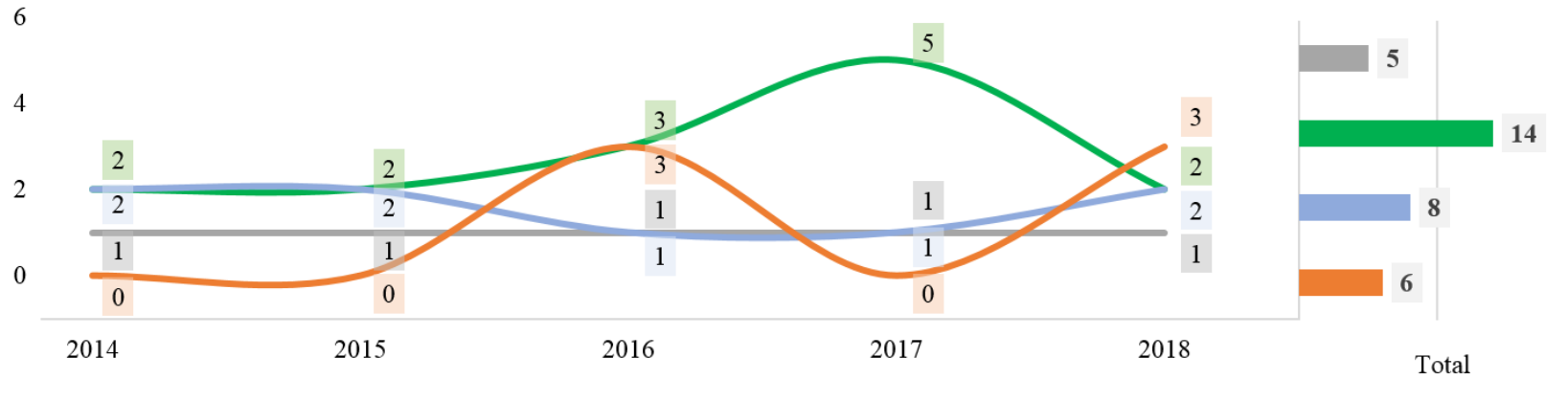

Teórico/Conceitual Proposição de método/modelo

Abordagem tecnológica aplicada

Estudos empíricos Fonte: Elaborado pelo autor.

Nas subseções seguintes abordou-se cada perspectiva a partir da discussão dos estudos analisados, com o objetivo de destacar os avanços recentes da área, assim como tendências observadas nos estudos do ponto de vista cientifico e prático.

\subsubsection{Estudos teóricos e conceituais}

A incorporação de tecnologias loT em sistemas logísticos é discutida por diversos autores, contemplando aspectos teóricos e conceituais, assim como analisando requisitos para implementação de tais tecnologias. Zou, Chen, Uysal e Zheng (2014) discutiram conceitos relacionados a aplicação de RFID em sistemas logísticos de alimentos e destacaram a tecnologia como promissora para viabilizar interconectividade e inteligência sistêmica na logística. Guo e Qu (2015) analisaram conceitualmente a influência da loT e computação em nuvem no contexto da "big data" em sistemas logísticos inteligentes, os autores discutem três arquiteturas de sistemas para integração dessas ferramentas. Maslarić, Nikoličić e Mirčetić (2016) apresentam uma análise do impacto da Indústria 4.0 na logística, apontando o conceito de "Physical Internet" como uma resposta as mudanças na indústria, em que o foco logístico será em padronização e interconectividade apoiada por tecnologias loT. Qu et al. (2017) analisaram a aplicação de modelos de dinâmicas de sistemas no contexto da adoção de loT em sistemas logísticos e de produção, fornecendo um roteiro de implementação e análise para integração de informações industriais e de transporte. Tu, Lim e Yang (2018a) propõem uma estrutura teórica para implementação de loT em produção logística e cadeias de suprimentos, os autores discutem os requisitos conceituais e contextos de aplicação, enfatizando o setor de manufatura discreta como promissor para a adoção de IoT. De uma forma geral, conceitualmente a área apresenta amadurecimento quanto a adoção de loT na logística, os autores nessa perspectiva apresentam direções convergentes do ponto de vista teórico com recomendações voltadas a prática. 


\subsubsection{Proposição de novos métodos e modelos para tomada de decisão}

Tecnologias loT auxiliam na coleta de dados em tempo real e controle adaptativo de sistemas, nessa direção os estudos classificados na perspectiva "proposição de novos métodos e modelos para tomada de decisão" no contexto logístico apresentaram uma grande variedade de abordagens para formalizar, estruturar e roteirizar a adoção de tecnologias loT considerando diversos aspectos de sistemas logísticos e sua interface com a produção. Nos estudos de Guo, Zhang, Zhao e Song (2017) e Zhang, Guo, Lv e Liu (2018) é abordada a integração produção e logística a partir de tecnologias loT, nessa mesma direção Chen, Chen e Hsu (2014), Qiu, Luo, Xu, Zhong e Huang (2015), Yang, Li e Hu (2016) e Trappey et al. (2017) discutem a proposição de abordagens considerando a integração de serviços logísticos com utilização de tecnologias loT, destacando como foco a capacidade de acompanhamento em tempo real das operações, Trappey et al. (2017) propõe um roteiro para implementação de um sistema logístico inteligente voltado a serviços.

Kong, Fang, Luo e Huang (2015) e Lee, Lv, Ng, Ho e Choy (2018) desenvolveram abordagens voltadas a gestão de armazéns e sistemas de distribuição, em ambos estudos é proposto a aplicação de IOT para controle em tempo real com capacidade adaptativa. Zhong, Lan, Xu, Dai e Huang (2016), Cho e Kim (2017) e Yan (2017) abordam a incorporação de dispositivos de RFID, propondo essa tecnologia em um modelo de gerenciamento em tempo real. Outro contexto observado nos estudos tratou da proposição de abordagens voltada para produtos alimentícios perecíveis, Yan (2017) e Zhang, Zhao e Qian (2017) apresentaram propostas para incorporações de loT nesse contexto.

Aplicações em contextos específicos foram propostas por He e Chu (2014) que tratou da utilização de loT para gestão da informação voltada a logística marítima, assim como Thürer et al. (2016) que propôs uma abordagem para logística reversa de resíduos sólidos considerando a incorporação de tecnologias IOT. De uma forma geral, nessa perspectiva os estudos se apoiaram em métodos de simulação para evidenciar a proposição dos métodos e modelos propostos, em alguns casos utilizando dados reais para testar demonstrar ganhos de performance, porém não evidenciando ainda uma aplicação direta e empírica das abordagens propostas.

\subsubsection{Desenvolvimento de abordagens tecnológicas aplicadas}

Nessa perspectiva foram classificados estudos com foco em desenvolvimento tecnológico considerando loT em sistemas logísticos, abordando tanto desenvolvimento computacional (e.g. softwares e sistemas) quanto de dispositivos. Os estudos de Li, Peng, Zhang, Wei e Li (2015), Kim, Lee e Oh (2015) e Kang, Park e Youm (2016) propuseram sistemas de rastreamento em tempo real, especificando dispositivos de sensoriamento e propondo ferramentas computacionais. Nessa mesma direção Chuang et al. (2017) desenvolveu um estudo para monitoramento em tempo real utilizando 
tecnologias wireless e bluetooth, os autores realizam uma prototipagem da abordagem proposta demonstrando a aplicabilidade. Outro estudo a realização prototipagem foi realizado por Chandra e Lee (2014) que desenvolveram uma arquitetura de transmissão de dados para monitoramento da temperatura e humidade no transporte de alimentos.

Os estudos desenvolvidos por Verdouw, Robbemond, Verwaart, Wolfert e Beulens (2018) e Accorsi, Cholette, Manzini e Tufano (2018) também tiveram foco em logística de alimentos, ambos desenvolveram arquiteturas para gerenciamento de informações na cadeia de suprimentos apoiados em tecnologias IoT. Em um contexto mais genérico, Chen, Zhao e Zhai (2014) desenvolveram um sistema para computação em nuvem utilizando dispositivos RFID para controle e monitoramento da cadeia de suprimentos.

Nessa perspectiva foi possível evidenciar um amadurecimento da área de pesquisa, em que se identificou a transição dos conceitos teóricos e proposição de modelos em direção ao desenvolvimento tecnológico da área.

\subsubsection{Estudos empíricos}

Aplicações de loT na logística considerando casos reais são reportados na literatura ainda com pouca frequência, no portfólio bibliográfico analisado foram classificados estudos com essas características. Tu (2018) realizou um levantamento tipo survey em Taiwan considerando a adoção de IoT no contexto da produção e logística, o autor utilizou sistema de equações estruturais para analisar as informações levantadas indicando que fatores como custo, pressões externas e a percepção de benefícios influencia a adoção de loT. Nessa direção Hu, Huang, Hou, Chen e Bulysheva (2016) utilizou analise fatorial exploratória para analisar na China a percepção dos clientes, concluindo que tecnologias IoT vão melhorar a relação empresa cliente.

Estudos de caso envolvendo loT e sistemas logísticos foram desenvolvidos por Hopkins \& Hawking (2018) que teve como foco a influência de adoção de loT na segurança e custos operacionais no transporte de caminhões. Tu, Lim e Yang (2018b) realizaram um estudo experimental para implementação de tecnologia loT em um sistema industrial complexo, envolvendo transporte e logística, encontrando resultados direcionados a maior flexibilize e controle. Qu et al. (2016) realizaram um estudo de caso com foco em sincronização da produção e logística demonstrando efetividade relacionada a adoção de IoT nesse contexto. Papert, Rimpler e Pflaum (2016) analisaram o setor farmacêutico considerando múltiplos estudos de caso na Alemanha, indicando a adoção de loT como solução para gerenciamento de cadeias logísticas complexas. 


\section{Conclusão}

Este artigo apresentou uma revisão sistemática exploratória abrangendo a aplicação de tecnologia loT em sistemas logísticos. Algumas limitações deste estudo podem ser apontadas, como: (i) Apenas duas bases cientificas foram consideradas, apesar de serem bases relevantes, no futuro estudos mais amplos podem incluir outros repositórios; (ii) Apenas artigos em inglês foram considerados, em estudos futuros para identificar desenvolvimentos locais podem ser adicionados também estudos em língua portuguesa; (iii) Apenas artigos recentes foram analisados, apesar de ser um dos objetivos desse estudo apenas considerar publicações recentes para fornecer um portfólio atualizado, um período maior de análise pode fornecer novas achados de pesquisa.

Foi possível responder as questões de pesquisas propostas, identificando um crescimento recente da área, grande contribuição de pesquisas por parte de instituições localizadas na China, Taiwan e Coreia do Sul, assim como importantes veículos de comunicação científica, destacando o Industrial Manufacturing \& Dara Systems e International Journal of Production Economics. Quanto as temáticas, as pesquisas investigadas relacionavam principalmente aspectos de tecnologias de RFID com estudos de arquiteturas de redes logísticas distribuídas com foco em gerenciamento e controle. As perspectivas identificadas permitem evidenciar o amadurecimento da adoção de loT na logística com um direcionamento recente para proposição de novos métodos e modelos em conjunto com estudos aplicados, indicando um foco em avaliar a teoria desenvolvida em aplicações reais.

Algumas lacunas foram evidenciadas e são apresentadas como direcionamento para futuras pesquisas, destacando três principais tópicos de interesse:

i. Evidenciação de um modelo genérico para adoção de loT em sistemas logísticos: as diversas abordagens encontradas apresentam convergência na maior parte dos aspectos, no entanto foi observado uma lacuna a partir dos estudos analisados, em que não foi encontrado um modelo genérico para aplicação de loT em sistemas logísticos. Frameworks para implementação de tecnologias loT em conjunto com desenvolvimento de um roadmap genérico a sistemas de transporte, armazenagem e distribuição são importantes tópicos a serem investigados no futuro, a fim de consolidar e integrar aspectos técnicos e operacionais relacionados a loT no contexto logístico.

ii. Integração produção e logística: Aplicações recorrentes de loT para integração da produção e logística podem fornecer plataformas de alta qualidade para decisões conjuntas com foco no controle abrangente de operações no contexto industrial. Essa é uma área emergente, possibilitada pela entrada de novas tecnologias como loT e demais aspectos relacionados a indústria 4.0, estudos 
nessa direção podem auxiliar o desenvolvimento de abordagens de sincronização e integração de cadeias de suprimentos.

iii. Monitoramento de dados em tempo real: controle efetivo do material e informação através da coleta de dados em tempo real ao longo do transporte e manuseio. Relacionando modelos de simulação, otimização e análise de dados em contextos reais com evidenciação empírica. A adoção de tecnologias 4.0 para monitoramento em tempo real ainda está em desenvolvimento tecnológico e conceitual, em que foi observado tanto nos estudos práticos quanto teóricos o amadurecimento desse conceito no controle de operações, dessa forma essa é uma importante lacuna a ser explorada como direção futura de pesquisa.

\section{Referências}

Accorsi, R., Cholette, S., Manzini, R., \& Tufano, A. (12 de 2018). A hierarchical data architecture for sustainable food supply chain management and planning. Journal of Cleaner Production, 203, 10391054. https://doi.org/10.1016/j.jclepro.2018.08.275.

Aria, M., \& Cuccurullo, C. (11 de 2017). bibliometrix : An R-tool for comprehensive science mapping analysis. Journal of Informetrics, 11, 959-975. https://doi.org/doi:10.1016/j.joi.2017.08.007.

Aryal, A., Liao, Y., Nattuthurai, P., \& Li, B. (12 de 2018). The emerging big data analytics and loT in supply chain management: a systematic review. Supply Chain Management: An International Journal. https://doi.org/10.1108/scm-03-2018-0149.

Avventuroso, G., Silvestri, M., \& Frazzon, E. M. (2018). Additive Manufacturing Plant for Large Scale Production of Medical Devices: A Simulation Study. IFAC-PapersOnLine, 51, 1442-1447. https://doi.org/10.1016/j.ifacol.2018.08.312.

Chandra, A. A., \& Lee, S. R. (10 de 2014). A Method of WSN and Sensor Cloud System to Monitor Cold Chain Logistics as Part of the loT Technology. International Journal of Multimedia and Ubiquitous Engineering, 9, 145-152. https://doi.org/10.14257/ijmue.2014.9.10.15.

Chen, S.-L., Chen, Y.-Y., \& Hsu, C. (3 de 2014). A New Approach to Integrate Internet-of-Things and Software-as-a-Service Model for Logistic Systems: A Case Study. Sensors, 14, 6144-6164. https://doi.org/10.3390/s140406144.

Chen, Y., Zhao, S., \& Zhai, Y. (2014). Construction of intelligent logistics system by RFID of internet of things based on cloud computing. Journal of Chemical and Pharmaceutical Research, 6, 1676-1679.

Cho, S., \& Kim, J. (3 de 2017). Smart Logistics Model on Internet of Things Environment. Advanced Science Letters, 23, 1599-1602. https://doi.org/10.1166/asl.2017.8604.

Chuang, C.-H., Lee, D.-H., Chang, W.-J., Weng, W.-C., Shaikh, M. O., \& Huang, C.-L. (4 de 2017). RealTime Monitoring via Patch-Type Piezoelectric Force Sensors for Internet of Things Based Logistics. IEEE Sensors Journal, 17, 2498-2506. https://doi.org/10.1109/jsen.2017.2665653. 
Cobo, M. J., López-Herrera, A. G., Herrera-Viedma, E., \& Herrera, F. (1 de 2011). An approach for detecting, quantifying, and visualizing the evolution of a research field: A practical application to the Fuzzy Sets Theory field. Journal of Informetrics, 5, 146-166.

https://doi.org/10.1016/j.joi.2010.10.002.

Frazzon, E. M., Kück, M., \& Freitag, M. (2018). Data-driven production control for complex and dynamic manufacturing systems. CIRP Annals, 67, 515-518.

https://doi.org/10.1016/j.cirp.2018.04.033.

Fruchterman, T. M., \& Reingold, E. M. (11 de 1991). Graph drawing by force-directed placement. Software: Practice and Experience, 21, 1129-1164. https://doi.org/10.1002/spe.4380211102.

Guerrero-Bote, V. P., \& Moya-Anegón, F. (10 de 2012). A further step forward in measuring journals' scientific prestige: The SJR2 indicator. Journal of Informetrics, 6, 674-688.

https://doi.org/10.1016/j.joi.2012.07.001.

Guo, Y., \& Qu, J. (8 de 2015). Study on Intelligent Logistics Management Information System Based on IOT and Cloud Computation in Big Data Era. The Open Cybernetics \& Systemics Journal, 9, 934-941. https://doi.org/10.2174/1874110x01509010934.

Guo, Z., Zhang, Y., Zhao, X., \& Song, X. (3 de 2017). A Timed Colored Petri Net Simulation-Based SelfAdaptive Collaboration Method for Production-Logistics Systems. Applied Sciences, 7, 235. https://doi.org/10.3390/app7030235.

Haq, A., \& Kannan, G. (2006). Effect of forecasting on the multi-echelon distribution inventory supply chain cost using neural network, genetic algorithm and particle swarm optimisation. International Journal of Services Operations and Informatics, 1, 1-22. https://doi.org/10.1504/IJSOI.2006.010186.

He, W., \& Chu, X. (2 de 2014). A Novel Navigation Information Management System for Food Maritime Logistics Based on Internet of Things. Advance Journal of Food Science and Technology, 6, 280-283. https://doi.org/10.19026/ajfst.6.25.

Heger, J., Grundstein, S., \& Freitag, M. (11 de 2017). Online-scheduling using past and real-time data. An assessment by discrete event simulation using exponential smoothing. CIRP Journal of Manufacturing Science and Technology, 19, 158-163. https://doi.org/10.1016/j.cirpj.2017.07.003.

Hopkins, J., \& Hawking, P. (5 de 2018). Big Data Analytics and loT in logistics: a case study. The International Journal of Logistics Management, 29, 575-591. https://doi.org/10.1108/ijlm-05-20170109.

Hu, M., Huang, F., Hou, H., Chen, Y., \& Bulysheva, L. (4 de 2016). Customized logistics service and online shoppers' satisfaction: an empirical study. (P. S. Professor Pan Wang, Ed.) Internet Research, 26, 484-497. https://doi.org/10.1108/intr-11-2014-0295.

Kang, Y.-S., Park, I.-H., \& Youm, S. (12 de 2016). Performance Prediction of a MongoDB-Based Traceability System in Smart Factory Supply Chains. Sensors, 16, 2126. https://doi.org/10.3390/s16122126.

Kim, J.-S., Lee, H.-J., \& Oh, R.-D. (5 de 2015). Smart Integrated Multiple Tracking System development for IOT based Target-oriented Logistics Location and Resource Service. International Journal of Smart Home, 9, 195-204. https://doi.org/10.14257/ijsh.2015.9.5.19. 
Kong, X. T., Fang, J., Luo, H., \& Huang, G. Q. (6 de 2015). Cloud-enabled real-time platform for adaptive planning and control in auction logistics center. Computers \& Industrial Engineering, 84, 79-90. https://doi.org/10.1016/j.cie.2014.11.005.

Lee, C. K., Lv, Y., Ng, K. K., Ho, W., \& Choy, K. L. (10 de 2017). Design and application of Internet of things-based warehouse management system for smart logistics. International Journal of Production Research, 56, 2753-2768. https://doi.org/10.1080/00207543.2017.1394592.

Lee, J., Bagheri, B., \& Kao, H.-A. (1 de 2015). A Cyber-Physical Systems architecture for Industry 4.0based manufacturing systems. Manufacturing Letters, 3, 18-23.

https://doi.org/10.1016/j.mfglet.2014.12.001.

Lee, Y. H., Jung, J. W., Eum, S. C., Park, S. M., \& Nam, H. K. (2006). Production quantity allocation for order fulfilment in the supply chain: a neural network based approach. Production Planning and Control, 17, 378-389. https://doi.org/10.1080/09537280600621909.

Leusin, M., Frazzon, E., Maldonado, M. U., Kück, M., \& Freitag, M. (11 de 2018). Solving the Job-Shop Scheduling Problem in the Industry 4.0 Era. Technologies, 6, 107. https://doi.org/10.3390/technologies6040107.

Li, Y. N., Peng, Y. L., Zhang, L., Wei, J. F., \& Li, D. (12 de 2015). Quality monitoring traceability platform of agriculture products cold chain logistics based on the internet of things. Chemical Engineering Transactions, 46, 517-522. https://doi.org/10.3303/CET1546087.

Lima-Junior, F. R., \& Carpinetti, L. C. (6 de 2019). Predicting supply chain performance based on SCOR ${ }^{\circledR}$ metrics and multilayer perceptron neural networks. International Journal of Production Economics, 212, 19-38. https://doi.org/10.1016/j.ijpe.2019.02.001.

Mahdavinejad, M. S., Rezvan, M., Barekatain, M., Adibi, P., Barnaghi, P., \& Sheth, A. P. (8 de 2018). Machine learning for internet of things data analysis: a survey. Digital Communications and Networks, 4, 161-175. https://doi.org/10.1016/j.dcan.2017.10.002.

Maslarić, M., Nikoličić, S., \& Mirčetić, D. (11 de 2016). Logistics Response to the Industry 4.0: the Physical Internet. Open Engineering, 6. https://doi.org/10.1515/eng-2016-0073.

Monostori, L., Kádár, B., Bauernhansl, T., Kondoh, S., Kumara, S., Reinhart, G., Sauer o., Schuh G., Sihn W., Ueda, K. (2016). Cyber-physical systems in manufacturing. CIRP Annals, 65, 621-641. https://doi.org/10.1016/j.cirp.2016.06.005.

Papert, M., Rimpler, P., \& Pflaum, A. (10 de 2016). Enhancing supply chain visibility in a pharmaceutical supply chain. International Journal of Physical Distribution \& Logistics Management, 46, 859-884. https://doi.org/10.1108/ijpdlm-06-2016-0151.

Peres, F. A., \& Fogliatto, F. S. (1 de 2018). Variable selection methods in multivariate statistical process control: A systematic literature review. Computers \& Industrial Engineering, 115, 603-619. https://doi.org/10.1016/j.cie.2017.12.006.

Qiu, X., Luo, H., Xu, G., Zhong, R., \& Huang, G. Q. (1 de 2015). Physical assets and service sharing for IoT-enabled Supply Hub in Industrial Park (SHIP). International Journal of Production Economics, 159, 4-15. https://doi.org/10.1016/j.ijpe.2014.09.001. 
Qu, T., Lei, S. P., Wang, Z. Z., Nie, D. X., Chen, X., \& Huang, G. Q. (5 de 2016). loT-based real-time production logistics synchronization system under smart cloud manufacturing. The International Journal of Advanced Manufacturing Technology, 84, 147-164. https://doi.org/10.1007/s00170-0157220-1.

Qu, T., Thürer, M., Wang, J., Wang, Z., Fu, H., Li, C., \& Huang, G. Q. (4 de 2017). System dyna mics analysis for an Internet-of-Things-enabled production logistics system. International Journal of Production Research, 55, 2622-2649. https://doi.org/10.1080/00207543.2016.1173738.

R Core Team. (2018). R: A Language and Environment for Statistical Computing. R Foundation for Statistical Computing. Fonte: https://www.R-project.org/.

Ray, P. P. (12 de 2016). A survey of loT cloud platforms. Future Computing and Informatics Journal, 1, 35-46. https://doi.org/10.1016/j.fcij.2017.02.001.

Silva, N., Ferreira, L. M., Silva, C., Magalhães, V., \& Neto, P. (2017). Improving Supply Chain Visibility With Artificial Neural Networks. Procedia Manufacturing, 11, 2083-2090. https://doi.org/10.1016/j.promfg.2017.07.329.

Tao, F., Qi, Q., Liu, A., \& Kusiak, A. (7 de 2018). Data-driven smart manufacturing. Journal of Manufacturing Systems, 48, 157-169. https://doi.org/10.1016/j.jmsy.2018.01.006.

Thoben, K.-D., Wiesner, S., \& Wuest, T. (2017). "Industrie 4.0" and smart manufacturing-a review of research issues and application examples. International Journal of Automation Technology, 11, 416. https://doi.org/10.20965/ijat.2017.p0004.

Thürer, M., Pan, Y. H., Qu, T., Luo, H., Li, C. D., \& Huang, G. Q. (12 de 2016). Internet of Things (IoT) driven kanban system for reverse logistics: solid waste collection. Journal of Intelligent Manufacturing. https://doi.org/10.1007/s10845-016-1278-y.

Trappey, A. J., Trappey, C. V., Fan, C.-Y., Hsu, A. P., Li, X.-K., \& Lee, I. J. (9 de 2017). loT patent roadmap for smart logistic service provision in the context of Industry 4.0. Journal of the Chinese Institute of Engineers, 40, 593-602. https://doi.org/10.1080/02533839.2017.1362325.

Tu, M. (2 de 2018). An exploratory study of Internet of Things (IOT) adoption intention in logistics and supply chain management. The International Journal of Logistics Management, 29, 131-151. https://doi.org/10.1108/ijlm-11-2016-0274.

Tu, M., Lim, M. K., \& Yang, M.-F. (2 de 2018). loT-based production logistics and supply chain system Part 1. Industrial Management \& Data Systems, 118, 65-95. https://doi.org/10.1108/imds-11-20160503.

Tu, M., Lim, M. K., \& Yang, M.-F. (2 de 2018). loT-based production logistics and supply chain system Part 2. Industrial Management \& Data Systems, 118, 96-125. https://doi.org/10.1108/imds-112016-0504.

Verdouw, C. N., Robbemond, R. M., Verwaart, T., Wolfert, J., \& Beulens, A. J. (8 de 2015). A reference architecture for loT-based logistic information systems in agri-food supply chains. Enterprise Information Systems, 12, 755-779. https://doi.org/10.1080/17517575.2015.1072643. 
Wang, L., Törngren, M., \& Onori, M. (10 de 2015). Current status and advancement of cyber-physical systems in manufacturing. Journal of Manufacturing Systems, 37, 517-527.

https://doi.org/10.1016/j.jmsy.2015.04.008.

Yan, R. (5 de 2017). Optimization approach for increasing revenue of perishable product supply chain with the Internet of Things. Industrial Management \& Data Systems, 117, 729-741. https://doi.org/10.1108/imds-07-2016-0297.

Yang, R., Li, B., \& Hu, Y. (5 de 2016). An Experimental Study for Intelligent Logistics: A Middleware Approach. Chinese Journal of Electronics, 25, 561-569. https://doi.org/10.1049/cje.2016.05.024.

Zhang, Y., Guo, Z., Lv, J., \& Liu, Y. (9 de 2018). A Framework for Smart Production-Logistics Systems Based on CPS and Industrial IOT. IEEE Transactions on Industrial Informatics, 14, 4019-4032. https://doi.org/10.1109/tii.2018.2845683.

Zhang, Y., Zhao, L., \& Qian, C. (10 de 2017). Modeling of an loT-enabled supply chain for perishable food with two-echelon supply hubs. Industrial Management \& Data Systems, 117, 1890-1905. https://doi.org/10.1108/imds-10-2016-0456.

Zhong, R. Y., Lan, S., Xu, C., Dai, Q., \& Huang, G. Q. (8 de 2015). Visualization of RFID-enabled shopfloor logistics Big Data in Cloud Manufacturing. The International Journal of Advanced Manufacturing Technology, 84, 5-16. https://doi.org/10.1007/s00170-015-7702-1.

Zou, Z., Chen, Q., Uysal, I., \& Zheng, L. (5 de 2014). Radio frequency identification enabled wireless sensing for intelligent food logistics. Philosophical Transactions of the Royal Society A: Mathematical, Physical and Engineering Sciences, 372, 20130313-20130313. https://doi.org/10.1098/rsta.2013.0313.

Apêndice A - Análise de conteúdo do portfólio bibliográfico (continua)

\section{Autores Objetivo}

Apresenta um estudo de caso de uma indústria de Qu et al. tintas que utiliza loT para (2016) resolver problemas de sincronização dos recursos logísticos

Propõe um modelo de negócios para suporte ao

Qiu et al.

(2015) compartilhamento de ativos físicos e serviços com ênfase na servitização da logística Zhong et al. caso de uma indústria de (2016)

Zou et al. (2014) tecnologia RFID e IoT Investiga diferentes aplicações de soluções de tecnologia RFID na logística

\section{Contexto de Aplicação}

Utiliza sensores RFID e computação em nuvem para disponibilizar acesso online e permitir o sincronismo das demandas de vendas, produção e os serviços públicos de logística na China

Através da adoção de tecnologia loT propõe o monitoramento em tempo real da utilização e disponibilidade de ativos físicos e serviços públicos de logística na China

Propõe modelo de abstração de dados para visualizar e interpretar padrões na logística produtiva com foco em aumento de performance

Propõe a utilização de tecnologia RFID com ênfase em redes de ultra banda larga para (iv) Estudo empírico

(ii) Proposição de método e/ou modelo

(ii) Proposição de método e/ou modelo da cadeia refrigerada 
(continuação)

\begin{tabular}{|c|c|c|c|c|}
\hline & & $\begin{array}{l}\text { monitoramento de produtos da } \\
\text { cadeia refrigerada }\end{array}$ & & \\
\hline $\begin{array}{l}\text { Chen, Chen } \\
\text { e Hsu } \\
\text { (2014) }\end{array}$ & $\begin{array}{l}\text { Propõe um modelo teórico } \\
\text { para desenvolvimento de } \\
\text { sistemas que permitem } \\
\text { integrar dispositivos, } \\
\text { processos e objetos }\end{array}$ & $\begin{array}{c}\text { Aborda o conceito de SaaS como } \\
\text { facilitador para integração } \\
\text { logística }\end{array}$ & $\begin{array}{l}\text { (ii) Proposição de } \\
\text { método e/ou } \\
\text { modelo }\end{array}$ & 19 \\
\hline $\begin{array}{l}\text { Qu et al. } \\
\text { (2017) }\end{array}$ & $\begin{array}{l}\text { Investiga a utilização da } \\
\text { dinâmica de sistemas para } \\
\text { desenvolvimento de } \\
\text { soluções de loT } \\
\text { economicamente viáveis }\end{array}$ & $\begin{array}{c}\text { Aborda simulações para } \\
\text { desenvolvimento e avaliação de } \\
\text { sistemas com base em RFID e } \\
\text { redes wireless de longo alcance } \\
\text { abrangendo as operações } \\
\text { internas e externas da logística de } \\
\text { produção }\end{array}$ & $\begin{array}{l}\text { (i) Teórico e/ou } \\
\text { conceitual }\end{array}$ & 17 \\
\hline $\begin{array}{l}\text { Kong et al. } \\
\text { (2015) }\end{array}$ & $\begin{array}{l}\text { Propõe uma arquitetura } \\
\text { para desenvolvimento de } \\
\text { soluções de loT com foco } \\
\text { nos desafios das vendas de } \\
\text { commodities e produtos } \\
\text { perecíveis }\end{array}$ & $\begin{array}{l}\text { Estudo de caso realizado em uma } \\
\text { empresa chinesa especializada na } \\
\text { venda de flores. Propõe soluções } \\
\text { para os desafios dos leilões de } \\
\text { produtos perecíveis considerando } \\
\text { a integração logística (informação } \\
\text { e movimentação dos produtos } \\
\text { em tempo hábil) }\end{array}$ & $\begin{array}{l}\text { (ii) Proposição de } \\
\text { método e/ou } \\
\text { modelo }\end{array}$ & 17 \\
\hline $\begin{array}{l}\text { Papert, } \\
\text { Rimpler e } \\
\text { Pflaum } \\
\text { (2016) }\end{array}$ & $\begin{array}{l}\text { Investiga a aplicação de } \\
\text { sensores RFID para } \\
\text { identificação e } \\
\text { monitoramento de produtos } \\
\text { na cadeia logística } \\
\text { farmacêutica }\end{array}$ & $\begin{array}{l}\text { Estudo de caso realizado com } \\
\text { empresas do ramo farmacêutico } \\
\text { alemão. Propõe modelo } \\
\text { conceitual para implementação } \\
\text { de um sistema para } \\
\text { monitoramento e identificação } \\
\text { dos produtos dentro da cadeia } \\
\text { logística farmacêutica }\end{array}$ & $\begin{array}{l}\text { (iv) Estudo } \\
\text { empírico }\end{array}$ & 9 \\
\hline $\begin{array}{l}\text { Hu et al. } \\
(2016)\end{array}$ & $\begin{array}{l}\text { Apresenta um estudo de } \\
\text { caso a respeito da } \\
\text { customização de serviços } \\
\text { logísticos e sua influência na } \\
\text { satisfação dos consumidores } \\
\text { de lojas de varejo online }\end{array}$ & $\begin{array}{l}\text { Propõe a utilização de tecnologia } \\
\text { loT para aumento de } \\
\text { performance na utilização de } \\
\text { serviços logísticos customizados e } \\
\text { consequente aumento da } \\
\text { satisfação do consumidor }\end{array}$ & $\begin{array}{l}\text { (iv) Estudo } \\
\text { empírico }\end{array}$ & 8 \\
\hline $\begin{array}{l}\text { Maslari, } \\
\text { Nikolii e } \\
\text { Mireti } \\
(2016)\end{array}$ & $\begin{array}{l}\text { Apresenta o conceito da } \\
\text { internet física e os desafios } \\
\text { na sua adoção pela indústria }\end{array}$ & $\begin{array}{l}\text { Abrange os desafios da cadeia de } \\
\text { suprimentos em sua totalidade } \\
\text { propondo a criação de modelos } \\
\text { de negócios sustentáveis em } \\
\text { relação trabalho humano, } \\
\text { segurança, padronização e } \\
\text { interoperabilidade dos serviços }\end{array}$ & $\begin{array}{l}\text { (i) Teórico e/ou } \\
\text { conceitual }\end{array}$ & 8 \\
\hline $\begin{array}{l}\text { Zhang, Guo } \\
\text { e Liu (2018) }\end{array}$ & $\begin{array}{l}\text { Apresentam um modelo } \\
\text { teórico para sistemas de } \\
\text { manufatura inteligente }\end{array}$ & $\begin{array}{l}\text { Propõe a utilização de tecnologia } \\
\text { IoT para aumento de } \\
\text { performance na utilização dos } \\
\text { recursos produtivos com foco em } \\
\text { economia de energia e redução } \\
\text { do tempo produtivo }\end{array}$ & $\begin{array}{l}\text { (ii) Proposição de } \\
\text { método e/ou } \\
\text { modelo }\end{array}$ & 7 \\
\hline $\begin{array}{l}\text { Guo et al. } \\
\text { (2017) }\end{array}$ & $\begin{array}{l}\text { Propõe um modelo para } \\
\text { desenvolvimento de redes } \\
\text { colaborativas auto } \\
\text { adaptáveis para otimização } \\
\text { logística e aumento de } \\
\text { performance econômica e }\end{array}$ & $\begin{array}{c}\text { Combina a utilização de } \\
\text { tecnologia RFID, computação em } \\
\text { nuvem, sensores, etc. para } \\
\text { criação de rede colaborativa com } \\
\text { foco em performance na logística } \\
\text { produtiva }\end{array}$ & $\begin{array}{l}\text { (ii) Proposição de } \\
\text { método e/ou } \\
\text { modelo }\end{array}$ & 7 \\
\hline
\end{tabular}


(continuação)

\begin{tabular}{|c|c|c|c|c|}
\hline & $\begin{array}{c}\text { ambiental na utilização de } \\
\text { recursos }\end{array}$ & & & \\
\hline Yan (2017) & $\begin{array}{c}\text { Propõe modelo de receitas } \\
\text { na cadeia de suprimentos de } \\
\text { pescados e aquicultura com } \\
\text { base na adoção de } \\
\text { tecnologia loT }\end{array}$ & $\begin{array}{l}\text { Estudo de caso realizado na China } \\
\text { compara as receitas antes e } \\
\text { depois da aplicação do modelo. } \\
\text { Ênfase na otimização dos custos } \\
\text { logísticos e diminuição de perdas } \\
\text { dos produtos perecíveis }\end{array}$ & $\begin{array}{l}\text { (ii) Proposição de } \\
\text { método e/ou } \\
\text { modelo }\end{array}$ & 7 \\
\hline $\begin{array}{l}\text { Kang, Park e } \\
\text { Youm } \\
\text { (2016) }\end{array}$ & $\begin{array}{c}\text { Aborda simulação e predição } \\
\text { de performance em sistemas } \\
\text { de rastreabilidade na } \\
\text { produção }\end{array}$ & $\begin{array}{l}\text { Utiliza tecnologia loT e Big Data } \\
\text { para simulação em sistemas de } \\
\text { rastreabilidade na cadeia de } \\
\text { suprimentos de peças de } \\
\text { automóveis }\end{array}$ & $\begin{array}{l}\text { (iii) Abordagem } \\
\text { tecnológica } \\
\text { aplicada }\end{array}$ & 7 \\
\hline $\begin{array}{l}\text { Verdouw et } \\
\text { al. (2018) }\end{array}$ & $\begin{array}{c}\text { Propõe uma arquitetura } \\
\text { para desenvolvimento de } \\
\text { soluções de loT com foco } \\
\text { nos desafios da cadeia de } \\
\text { suprimento do agronegócio } \\
\text { em especial dos produtos } \\
\text { perecíveis }\end{array}$ & $\begin{array}{l}\text { Combina tecnologias loT e } \\
\text { computação em nuvem para } \\
\text { solução de problemas de } \\
\text { planejamento na cadeia de } \\
\text { suprimentos centralizada e } \\
\text { descentralizada }\end{array}$ & $\begin{array}{l}\text { (iii) Abordagem } \\
\text { tecnológica } \\
\text { aplicada }\end{array}$ & 6 \\
\hline $\begin{array}{c}\text { Thürer et al. } \\
\text { (2016) }\end{array}$ & $\begin{array}{l}\text { Apresenta modelo teórico } \\
\text { para desenvolvimento de } \\
\text { sistema Kanban para } \\
\text { resolver os problemas na } \\
\text { demanda de serviços na } \\
\text { logística reversa }\end{array}$ & $\begin{array}{l}\text { Propõe utilizar um sistema } \\
\text { Kanban combinado com } \\
\text { tecnologia loT identificando os } \\
\text { pontos de demanda de coleta de } \\
\text { lixo (de natureza estocástica) e } \\
\text { sincronismo dos pontos de coleta } \\
\text { envolvendo grandes distância }\end{array}$ & $\begin{array}{l}\text { (ii) Proposição de } \\
\text { método e/ou } \\
\text { modelo }\end{array}$ & 6 \\
\hline $\begin{array}{l}\text { Accorsi et } \\
\text { al. (2018) }\end{array}$ & $\begin{array}{l}\text { Propõe um modelo para } \\
\text { criação de base de dados } \\
\text { para tomada de decisão com } \\
\text { foco em otimização logística } \\
\text { para o segmento de } \\
\text { produtos agrícolas }\end{array}$ & $\begin{array}{l}\text { Avalia o aumento da performance } \\
\text { dos serviços logísticos através de } \\
\text { um estudo de caso de uma } \\
\text { empresa italiana responsável pela } \\
\text { distribuição de frutas }\end{array}$ & $\begin{array}{l}\text { (iii) Abordagem } \\
\text { tecnológica } \\
\text { aplicada }\end{array}$ & 4 \\
\hline $\begin{array}{c}\text { Tu, Lim e } \\
\text { Yang (2018) }\end{array}$ & $\begin{array}{l}\text { Propõe um modelo teórico } \\
\text { para desenvolvimento de } \\
\text { sistemas distribuídos que } \\
\text { permitem integrar } \\
\text { dispositivos, processos e } \\
\text { objetos }\end{array}$ & $\begin{array}{l}\text { Otimização do fluxo de materiais } \\
\text { e fluxo de informação na gestão } \\
\text { da cadeia de suprimentos }\end{array}$ & $\begin{array}{l}\text { (i) Teórico e/ou } \\
\text { conceitual }\end{array}$ & 4 \\
\hline $\begin{array}{l}\text { Trappey et } \\
\text { al. (2017) }\end{array}$ & $\begin{array}{l}\text { Apresenta um estudo de } \\
\text { caso sobre o registro de } \\
\text { patentes envolvendo a } \\
\text { adoção de loT em serviços } \\
\text { logísticos }\end{array}$ & $\begin{array}{l}\text { Avalia o desenvolvimento e } \\
\text { adoção de tecnologias orientadas } \\
\text { ao conceito de loT bem como } \\
\text { apresenta tendências no registro } \\
\text { de patentes tecnológicas }\end{array}$ & $\begin{array}{l}\text { (ii) Proposição de } \\
\text { método e/ou } \\
\text { modelo }\end{array}$ & 4 \\
\hline $\begin{array}{l}\text { Chuang et } \\
\text { al. (2017) }\end{array}$ & $\begin{array}{c}\text { Utilização de sensores } \\
\text { piezoelétricos no } \\
\text { desenvolvimento de } \\
\text { aplicativos para prestadores } \\
\text { de serviços logísticos com o } \\
\text { propósito de aumentar a } \\
\text { segurança na entrega de } \\
\text { mercadorias }\end{array}$ & $\begin{array}{l}\text { Através de um sistema integrado } \\
\text { que combina sensores de pressão } \\
\text { e sistemas em nuvem, permite } \\
\text { rastreamento de carga e } \\
\text { acompanhamento em tempo real } \\
\text { de eventos como quedas ou } \\
\text { rompimentos de embalagens }\end{array}$ & $\begin{array}{l}\text { (iii) Abordagem } \\
\text { tecnológica } \\
\text { aplicada }\end{array}$ & 4 \\
\hline $\begin{array}{l}\text { Li et al. } \\
\text { (2015) }\end{array}$ & $\begin{array}{l}\text { Utilização de sensores de } \\
\text { temperatura e umidade para } \\
\text { monitoramento de }\end{array}$ & $\begin{array}{l}\text { Combina sensores, tecnologia } \\
\text { RFID e redes wireless de longo } \\
\text { alcance aumentando a } \\
\text { performance e segurança no }\end{array}$ & $\begin{array}{l}\text { (iii) Abordagem } \\
\text { tecnológica } \\
\text { aplicada }\end{array}$ & 4 \\
\hline
\end{tabular}


(continuação)

\begin{tabular}{|c|c|c|c|c|}
\hline & $\begin{array}{l}\text { transportes da cadeia } \\
\text { refrigerada }\end{array}$ & $\begin{array}{c}\text { monitoramente de cargas } \\
\text { sensíveis a variação de umidade } \\
\text { e/ou temperatura }\end{array}$ & & \\
\hline $\begin{array}{l}\text { Kim, Lee e } \\
\text { Oh (2015) }\end{array}$ & $\begin{array}{l}\text { Sugere o desenvolvimento } \\
\text { de um sistema integrado } \\
\text { para rastreamento nos } \\
\text { serviços de distribuição de } \\
\text { produtos }\end{array}$ & $\begin{array}{l}\text { Combina utilização de sensores } \\
\text { RFID, computação em nuvem e } \\
\text { GPS para aumentar eficiência no } \\
\text { carregamento e transporte de } \\
\text { produtos }\end{array}$ & $\begin{array}{l}\text { (iii) Abordagem } \\
\text { tecnológica } \\
\text { aplicada }\end{array}$ & 4 \\
\hline $\begin{array}{l}\text { Chandra e } \\
\text { Lee (2014) }\end{array}$ & $\begin{array}{l}\text { Utilização de sensores de } \\
\text { temperatura e umidade para } \\
\text { monitoramento de } \\
\text { transportes da cadeia } \\
\text { refrigerada }\end{array}$ & $\begin{array}{l}\text { Combina sensores, tecnologia } \\
\text { RFID e redes wireless de longo } \\
\text { alcance aumentando a } \\
\text { performance e segurança no } \\
\text { monitoramento de cargas } \\
\text { sensíveis a variação de umidade } \\
\text { e/ou temperatura }\end{array}$ & $\begin{array}{l}\text { (iii) Abordagem } \\
\text { tecnológica } \\
\text { aplicada }\end{array}$ & 4 \\
\hline Tu (2018) & $\begin{array}{c}\text { Aborda os fatores } \\
\text { determinantes para adoção } \\
\text { de tecnologia loT na logística }\end{array}$ & $\begin{array}{l}\text { Pesquisa realizada com indústrias } \\
\text { de Twaian apresenta fatores } \\
\text { como benefícios, custos e pressão } \\
\text { externa como determinantes } \\
\text { para adoção enquanto a } \\
\text { confiança na tecnologia ainda } \\
\text { tem efeito negativo }\end{array}$ & $\begin{array}{l}\text { (iv) Estudo } \\
\text { empírico }\end{array}$ & 3 \\
\hline $\begin{array}{l}\text { Hopkins e } \\
\text { Hawking } \\
\text { (2018) }\end{array}$ & $\begin{array}{l}\text { Utilização de sensores e } \\
\text { vídeo monitoramento no } \\
\text { desenvolvimento de } \\
\text { estratégia para prestadores } \\
\text { de serviços logísticos com o } \\
\text { propósito de aumentar a } \\
\text { segurança e performance no } \\
\text { transporte de mercadorias }\end{array}$ & $\begin{array}{c}\text { Estudo de caso combina } \\
\text { tecnologia loT e Big Data para } \\
\text { avaliar comportamento funcional } \\
\text { dos motoristas identificando } \\
\text { fadiga e padrões do serviço com } \\
\text { foco otimização de rotas e } \\
\text { abastecimento de combustível, } \\
\text { manutenção preditiva entre } \\
\text { outros }\end{array}$ & $\begin{array}{l}\text { (iv) Estudo } \\
\text { empírico }\end{array}$ & 3 \\
\hline $\begin{array}{l}\text { Lee et al. } \\
(2018)\end{array}$ & $\begin{array}{l}\text { Propõe um modelo para } \\
\text { desenvolvimento de sistema } \\
\text { com foco na otimização de } \\
\text { operações de armazém }\end{array}$ & $\begin{array}{l}\text { Combina utilização de sensores } \\
\text { RFID e lógica Fuzzy para } \\
\text { otimização das operações } \\
\text { internas em relação a } \\
\text { produtividade da coleta e } \\
\text { ocupação do espaço }\end{array}$ & $\begin{array}{l}\text { (ii) Proposição de } \\
\text { método e/ou } \\
\text { modelo }\end{array}$ & 3 \\
\hline $\begin{array}{l}\text { Tu, Lim e } \\
\text { Yang (2018) }\end{array}$ & $\begin{array}{c}\text { Aplicação e validação de } \\
\text { modelo de desenvolvimento } \\
\text { de sistemas distribuídos que } \\
\text { permitem integrar } \\
\text { dispositivos, processos e } \\
\text { objetos }\end{array}$ & $\begin{array}{l}\text { Estudo de caso aplicado em uma } \\
\text { fábrica de bicicletas elétricas em } \\
\text { Taiwan. Otimização do fluxo de } \\
\text { materiais e fluxo de informação } \\
\text { na logística produtiva com } \\
\text { aumento de performance no } \\
\text { manuseio e rastreabilidade dos } \\
\text { produtos no fluxo da linha de } \\
\text { montagem e acabamento }\end{array}$ & $\begin{array}{l}\text { (iv) Estudo } \\
\text { empírico }\end{array}$ & 3 \\
\hline $\begin{array}{l}\text { Zhang, Zhao } \\
\text { e Qian } \\
\text { (2017) }\end{array}$ & $\begin{array}{l}\text { Apresenta um modelo de } \\
\text { compartilhamento de } \\
\text { informações que utiliza loT } \\
\text { para resolver problemas de } \\
\text { sincronização dos recursos } \\
\text { logísticos na cadeia de } \\
\text { suprimentos de produtos } \\
\text { perecíveis }\end{array}$ & $\begin{array}{c}\text { Estudo de caso aborda a } \\
\text { utilização de áreas comuns e } \\
\text { compartilhamento de } \\
\text { informações referente } \\
\text { movimentação de produtos } \\
\text { perecíveis com foco no aumento } \\
\text { de eficiência em serviços públicos } \\
\text { de logística na China }\end{array}$ & $\begin{array}{l}\text { (ii) Proposição de } \\
\text { método e/ou } \\
\text { modelo }\end{array}$ & 3 \\
\hline
\end{tabular}


(conclusão)

\begin{tabular}{|c|c|c|c|c|}
\hline $\begin{array}{l}\text { Cho e Kim } \\
\text { (2017) }\end{array}$ & $\begin{array}{c}\text { Sugere a utilização de } \\
\text { tecnologia loT para criar um } \\
\text { sistema de monitoramento } \\
\text { do transporte de } \\
\text { mercadorias }\end{array}$ & $\begin{array}{c}\text { Com base na utilização de } \\
\text { sensores RFID enfatiza a } \\
\text { otimização do serviço com foco } \\
\text { em segurança e redução de } \\
\text { custos para entregas no endereço } \\
\text { e prazo corretos }\end{array}$ & $\begin{array}{l}\text { (ii) Proposição de } \\
\text { método e/ou } \\
\text { modelo }\end{array}$ & 3 \\
\hline $\begin{array}{l}\text { Yang, Li e } \\
\text { Hu (2016) }\end{array}$ & $\begin{array}{l}\text { Propõe o desenvolvimento } \\
\text { de uma camada de serviço } \\
\text { (middleware) capaz de } \\
\text { monitorar e coordenar } \\
\text { serviços logísticos }\end{array}$ & $\begin{array}{c}\text { Arquitetura é baseada em } \\
\text { algoritmos responsáveis por } \\
\text { designar, em tempo de execução, } \\
\text { serviços e componentes que } \\
\text { podem participar de uma } \\
\text { transação logística, otimizando a } \\
\text { performance dos recursos } \\
\text { envolvidos }\end{array}$ & $\begin{array}{l}\text { (ii) Proposição de } \\
\text { método e/ou } \\
\text { modelo }\end{array}$ & 3 \\
\hline $\begin{array}{c}\text { Guo e Qu } \\
\text { (2015) }\end{array}$ & $\begin{array}{c}\text { Avalia a utilização de } \\
\text { tecnologia loT e sua } \\
\text { aplicação no campo da } \\
\text { logística aplicada a geologia }\end{array}$ & $\begin{array}{l}\text { Aborda aspectos referente o } \\
\text { manuseio e gestão inteligente de } \\
\text { material geológico, com ênfase } \\
\text { ao desenvolvimento da indústria } \\
\text { de mineração, situações } \\
\text { emergenciais e direitos de } \\
\text { exploração de minério }\end{array}$ & $\begin{array}{l}\text { (i) Teórico e/ou } \\
\text { conceitual }\end{array}$ & 3 \\
\hline $\begin{array}{l}\text { He e Chu } \\
(2015)\end{array}$ & $\begin{array}{l}\text { Propõe a construção de um } \\
\text { sistema de navegação } \\
\text { marítima baseado no } \\
\text { compartilhamento de } \\
\text { informações }\end{array}$ & $\begin{array}{c}\text { Combina a utilização de } \\
\text { tecnologia loT, sensores e } \\
\text { computação em nuvem para cria } \\
\text { um sistema de navegação para } \\
\text { aumento de performance e } \\
\text { segurança na logística marítima } \\
\text { de alimentos }\end{array}$ & $\begin{array}{l}\text { (ii) Proposição de } \\
\text { método e/ou } \\
\text { modelo }\end{array}$ & 3 \\
\hline $\begin{array}{c}\text { Chen, Zhao } \\
\text { e Zhai } \\
\text { (2014) }\end{array}$ & $\begin{array}{c}\text { Avalia a utilização de } \\
\text { tecnologia loT para } \\
\text { monitoramento de veículos } \\
\text { de carga }\end{array}$ & $\begin{array}{l}\text { Combina a utilização de RFID e } \\
\text { computação em nuvem para } \\
\text { monitorar veículos disponíveis no } \\
\text { pátio, tempo de carga entre } \\
\text { outros, com foco no aumento de } \\
\text { performance nas operações de } \\
\text { armazém }\end{array}$ & $\begin{array}{l}\text { (iii) Abordagem } \\
\text { tecnológica } \\
\text { aplicada }\end{array}$ & \\
\hline
\end{tabular}

